\title{
SMith College
}

\section{Smith ScholarWorks}

$11-16-2007$

\section{Supercurrent Survival under a Rosen-Zener Quench of Hard-Core Bosons}

\author{
I. Klich \\ California Institute of Technology \\ Courtney Lannert \\ Wellesley College, clannert@smith.edu \\ G. Refael \\ California Institute of Technology
}

Follow this and additional works at: https://scholarworks.smith.edu/phy_facpubs

Part of the Physics Commons

\section{Recommended Citation}

Klich, I.; Lannert, Courtney; and Refael, G., "Supercurrent Survival under a Rosen-Zener Quench of HardCore Bosons" (2007). Physics: Faculty Publications, Smith College, Northampton, MA.

https://scholarworks.smith.edu/phy_facpubs/67

This Article has been accepted for inclusion in Physics: Faculty Publications by an authorized administrator of Smith ScholarWorks. For more information, please contact scholarworks@smith.edu 


\title{
Supercurrent Survival under a Rosen-Zener Quench of Hard-Core Bosons
}

\author{
I. Klich, ${ }^{1}$ C. Lannert, ${ }^{2}$ and G. Refael ${ }^{1}$ \\ ${ }^{1}$ Department of Physics, California Institute of Technology, MC 114-36 Pasadena, California 91125, USA \\ ${ }^{2}$ Department of Physics, Wellesley College, Wellesley, Massachusetts 02481, USA
}

(Received 29 June 2007; published 16 November 2007)

\begin{abstract}
We study the survival of supercurrents in a system of impenetrable bosons on a lattice, subject to a quantum quench from its critical superfluid phase to an insulating phase. We show that the evolution of the current when the quench follows a Rosen-Zener profile is exactly solvable. This allows us to analyze a quench of arbitrary rate, from a sudden destruction of the superfluid to a slow opening of a gap. The decay and oscillations of the current are analytically derived and studied numerically along with the momentum distribution after the quench. In the case of small supercurrent boosts $\nu$, we find that the current surviving at long times is proportional to $\nu^{3}$.
\end{abstract}

PACS numbers: 67.40.Fd, 03.75.Kk, 05.70.Ln

Progress in the field of experimental cold atom systems allows a controlled and direct access to the nonequilibrium physics of quantum many body systems. This becomes particularly exciting when the system is in the vicinity of a phase transition and is driven through it as in the case of the superfluid(SF)-insulator transition [1], or the magnetic ordering transition [2]. In such situations, complex physics is often exhibited; a prominent example is defect formation in the ordered phase due to a fast quench [3], which is qualitatively understood using the Kibble-Zurek mechanism [4] originally proposed in cosmology to describe domain formation during cooling of the universe. Especially interesting are low-dimensional quantum systems under a "quantum quench," with a Hamiltonian driven through a quantum-critical point. This can be achieved in quantum gases confined in highly anisotropic traps and in optical lattices [5,6]. For instance, it was recently demonstrated that when a system is driven from an ordered to a disordered phase, the order-parameter correlations experience "revival" $[1,7-10]$. In the special case of driving an off-critical $1 d$ system into criticality, Calabrese and Cardy [11] showed that correlation functions can be expressed using correlations of the final critical state. Despite these advances, as well as qualitative understanding of "revival" phenomena and the KibbleZurek mechanism, analytical and exact results in this field are scarce.

Here, we focus on a system driven out of criticality by varying an external field. In practice, this is a generic case, but elegant general results as in Ref. [11] for the opposing case are mostly absent. Special cases that have previously been studied analytically are the behavior of a dipole model of a Mott insulator in an external electric field when the field is suddenly changed from a "no dipole" state to a maximally polarized state [12], and the dynamics of traversing spin chains between two phases [7]. A related work, Ref. [8], describes bosons driven abruptly from a Mott to superfluid phase and showed collective oscillations of the superfluid order parameter with period proportional to the gap in the initial Mott state. In [13], an SF to Mott quench was analyzed within a mean field approach. In [10], the evolution of hard-core bosons (HCBs) undergoing a quantum quench was studied numerically.

A natural question arising in the SF-insulator transition regards the fate of supercurrents in the system. In this Letter, we describe the evolution of supercurrents under quenching of a 1-d lattice gas of HCBs, the so called Tonks-Girardeu gas $[14,15]$, from SF to insulator. Such systems may be formed by bosons at low temperatures and densities [16]. Recently, a system of HCBs in an optical lattice has been realized in an ultracold dilute gas [6]. We calculate the current as a function of time, while concentrating on the long-time current survival rate. Our analysis reveals fast, Bloch-like, oscillations that are superimposed on a decay and a slower envelope function. The surviving current is found to be proportional to $\nu^{3}$, where $\nu$ is the initial supercurrent. Note that the decay of supercurrents was considered before in Ref. [9] close to the Mottsuperfluid transition. In addition, we present numerics for the evolution of the momentum distribution.

Our study relies on a generalization of the Rosen-Zener problem [17] to the context of HCBs. The Rosen-Zener problem describes a spin evolving in a time-dependent magnetic field with a particular profile, where an $x-y$ magnetic field (analogous to a gap) is turned on while a $z$-field remains constant. In contrast, Landau-Zener dynamics describe a spin in a constant $x$-y field, with $B_{z}$ swept through zero; thus, it is useful for describing traversing the system through a quantum critical point [7]. The integrability of the Rosen-Zener evolution allows us to probe sudden quench dynamics as well as the response to a finite quenching time and so goes beyond previous treatments, which have dealt with an abrupt quench.

The system we consider has the Hamiltonian

$$
H=-w \sum\left(b_{i}^{\dagger} b_{i+1}+\text { H.c. }\right)+V(t) \sum(-1)^{i} b_{i}^{\dagger} b_{i}
$$

with $b_{i}^{\dagger}$ a boson creation operator at site $i$ which obeys $\left(b_{i}^{\dagger}\right)^{2}=0$, thus imposing the impenetrability of the HCBs. 
Throughout, we work in units such that the lattice spacing is $1 . V(t)$ is the amplitude of an externally applied potential, which tunes the system from its superfluid phase at $V=0$, to an insulating phase at $V \neq 0$. When $V(t)$ has the Rosen-Zener shape, this evolution can be solved exactly. The Rosen-Zener profile is given by

$$
V(t)= \begin{cases}V_{0} \frac{1}{\cosh \frac{\pi t}{T}} ; & t<0 \\ V_{0} ; & t \geq 0\end{cases}
$$

where $T$ is the turning-on, or quenching time.

The HCB problem is equivalent to an infinite system of spins precessing in a time-dependent magnetic field. The evolution of a spin in the $\hat{z}$ direction under $B_{x}=$ $V(t)$ given in Eq. (2) (Fig. 1) was solved exactly by Rosen and Zener [17]. This exact nonequilibrium evolution allows us to establish the state of the system at $t=0$, from which the system is evolved by the final Hamiltonian. We make use of this evolution to analyze the fate of a supercurrent, introduced at $t \rightarrow-\infty$ [when the system is in the gapless SF phase and $V(-\infty)=0]$. At $t \rightarrow-\infty$, it is convenient to regard a current carrying state as the ground state of the HCB Hamiltonian observed from a moving frame, which corresponds to the boosted Hamiltonian $-w \sum\left(e^{i \nu} b_{i}^{\dagger} b_{i+1}+\right.$ H.c. $)$. Here, $\nu$ is the "boost" wave vector, which shifts modes with momentum $k$ to $k+\nu . \nu$ is related to the current at $t \rightarrow-\infty$ by $j(t=-\infty)=$ $(2 w / \pi) \sin (\nu)$.

The first step in our analysis applies the Wigner-Jordan transformation to the HCB operators [18], $b_{i}^{\dagger}=$ $e^{\sum_{j<i} a_{j}^{\dagger} a_{j}} a_{i}^{\dagger}$, with $a_{j}$ being a fermion operator associated with site $j$. In the "fermion" picture, the presence of the supercurrent is expressed by choosing the "Fermi sea" of the $a$ fermions to be shifted in momentum. That is, the $k$ occupation of the fermions is

$$
n_{s}(k)=\Theta\left(-k_{F}+\nu<k<k_{F}+\nu\right),
$$

where $k_{F}$ is the Fermi momentum. Let us now assume half filling, so $k_{F}=\pi / 2$. The current density is given by

$$
j=\frac{i w}{L} \sum_{l}\left\langle b_{l+1}^{\dagger} b_{l}-\text { H.c. }\right\rangle
$$

where $L$ is the total number of sites.

To find the evolution of the current, we first address the evolution of the fermion operators $a_{j}$ introduced above. By rewriting the Hamiltonian as

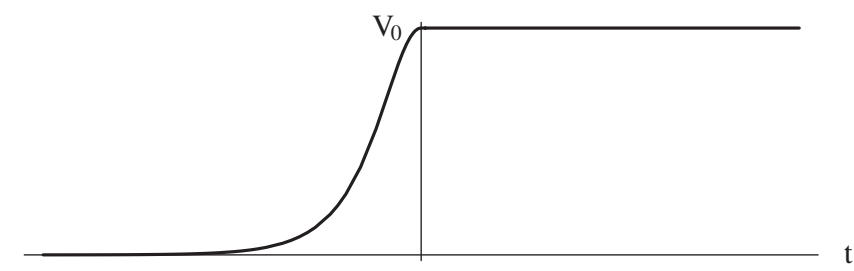

FIG. 1. The Rosen-Zener superlattice switch-on profile, $V(t)$.

$$
\begin{aligned}
& H_{0}=-\sum_{k<|\pi|} 2 w \cos (k) a_{k}^{\dagger} a_{k} \\
& H_{d}=\frac{V(t)}{2} \sum_{k<|\pi|}\left(a_{k}^{\dagger} a_{k+\pi}+a_{k+\pi}^{\dagger} a_{k}\right),
\end{aligned}
$$

we note that $k$ couples to $k+\pi$ (or equivalently $k-\pi$ since $k$ is the same up to multiples of $2 \pi$ ). Thus, we can write the Hamiltonian as

$$
\begin{aligned}
H(t) & =\otimes_{|k|<(\pi / 2)} H_{k}(t) \\
\text { with } \quad H_{k}(t) & =2 w \cos (k) \sigma_{z}-V(t) \sigma_{x}
\end{aligned}
$$

acting in the $\left\{a_{k}^{\dagger}, a_{k+\pi}^{\dagger}\right\}$ mode space, and explicitly breaking the problem into a product of noninteracting spin systems. Thus, solving the evolution of the HCB system is equivalent to solving for the time evolution of an infinite series of time-dependent two-level systems.

For a given $k$, the Schrödinger equation generated by Eq. (6) for a fermion operator $\psi^{\dagger}(t)=s(t) a_{k}^{\dagger}+p(t) a_{k+\pi}^{\dagger}$ can be rewritten as the second order differential equation:

$$
\ddot{S}=-\frac{V_{0}^{2}}{\cosh \left(\frac{\pi t}{T}\right)^{2}} S+\left[4 i w \cos (k)-\frac{\pi}{T} \tanh \left(\frac{\pi t}{T}\right)\right] \dot{S}
$$

for $S(t)=e^{2 i w \cos (k) t} s(t)$, and the same equation for $P(t)=$ $e^{-2 i w \cos (k) t} p(t)$, with $w \rightarrow-w$. This equation, via a change of variable $z=\frac{1}{2}\left[\tanh \left(\frac{\pi t}{T}\right)+1\right]$, can be recast into a hypergeometric differential equation. The solution, satisfying the initial condition $|S(-\infty)|=1,|P(-\infty)=0|$ (recall $|k|<\frac{\pi}{2}$ ), is given in terms of the hyper-geometric function:

$$
\begin{aligned}
S(z)= & { }_{2} F_{1}(\alpha,-\alpha, c ; z) \\
P(z)= & -i \sqrt{z(1-z)} \frac{2 \pi \alpha^{2} e^{-4 i w \cos (k) t}}{c T} \\
& \times{ }_{2} F_{1}(1+\alpha, 1-\alpha, 1+c ; z)
\end{aligned}
$$

where [19] $\alpha=\frac{V_{0} T}{\pi} ; c=\frac{1}{2}-\frac{2 i w T \cos (k)}{\pi}$. We now use the solution at $t=0$ [setting $z \rightarrow \frac{1}{2}$ in (8)] as a boundary condition for the dynamics under the final Hamiltonian.

The evolution of the $a_{j}$ 's at times $t>0$ is obtained by diagonalizing Eq. (6) at fixed $V=V_{0}$. This results in

$$
\begin{aligned}
a_{k}^{\dagger}(t) & =A_{k} a_{k}^{\dagger}(0)+B_{k} a_{k+\pi}^{\dagger}(0) \\
a_{k+\pi}^{\dagger}(t) & =\overline{A_{k}} a_{k+\pi}^{\dagger}(0)-B_{k} a_{k}^{\dagger}(0)
\end{aligned}
$$

where $A, B$ are given explicitly by the relations

$$
A_{k}=\cos \left(E_{k} t\right)+i \cos \theta \sin \left(E_{k}\right) \quad B_{k}=i \sin \theta \sin \left(E_{k} t\right)
$$

with

$$
-\pi<k<\pi, \quad E_{k}=\sqrt{4 w^{2} \cos ^{2} k+V_{0}^{2}}
$$

and $\theta$ is defined through $\tan \theta=-\frac{V_{0}}{2 w \cos k}$.

Using Eq. (8) with $z \rightarrow 1 / 2$ to find $a_{k}^{\dagger}(0), a_{k+\pi}^{\dagger}(0)$ and substituting in Eq. (4), we find 


$$
\begin{aligned}
\langle j(t)\rangle= & 4 w \int_{-\pi / 2}^{\pi / 2} \frac{d k}{2 \pi} \sin (k)\left(\left|s_{0} A_{k}-p_{0} B_{k}^{*}\right|^{2}-\frac{1}{2}\right) \\
& \times\left(n_{k}-n_{k+\pi}\right)
\end{aligned}
$$

where $n_{k}$ is the fermion momentum (Fermi-Dirac) occupation: $\left\langle a_{k}^{\dagger} a_{k^{\prime}}\right\rangle=\delta_{k k^{\prime}} n_{k}$. The above Eqs. (8)-(12) constitute a complete description of current evolution under a Rosen-Zener quench.

We first concentrate on an initial state with a supercurrent at half filling and with a short switching time, $w T \nu \ll 1$, which allows nonadiabatic transitions. In this case, to leading order near $k \sim \pi / 2$, we may take $c \sim 1 / 2$, $\Gamma(c) \approx \Gamma(1 / 2)=\sqrt{\pi}$, giving

$$
p(0)=-i \sin \left(\frac{V_{0} T}{2}\right) \quad s(0)=\cos \left(\frac{V_{0} T}{2}\right) .
$$

Assuming small supercurrents $(\nu \ll 1)$, the current evolution, Eq. (12), simplifies to

$$
\langle j\rangle=\frac{2 w \nu}{\pi} \mathcal{M}\left[(2 t-T) V_{0}, \kappa \nu \sqrt{t}\right]
$$

where

$$
\mathcal{M}(x, y)=\frac{\cos (x) \mathcal{C}(y)-\sin (x) \mathcal{S}(y)}{y}
$$

and $\mathcal{S}, \mathcal{C}$ are the Fresnel functions $\mathcal{S}(z)=\int_{0}^{z} d u \sin \left(\frac{\pi}{2} u^{2}\right)$, $\mathcal{C}(z)=\int_{0}^{z} d u \cos \left(\frac{\pi}{2} u^{2}\right)$, and $\kappa=\sqrt{\frac{8 w^{2}}{\pi V_{0}}}$.

Figure 2 shows the current evolution for an abrupt quench. The evolution is characterized by fast oscillations superimposed on a slow envelope which oscillates and decays. The time between successive maxima of the slow envelope can be estimated by looking at the extrema of the Fresnel integrals in Eq. (15). From $\partial_{z} \mathcal{C}(z)=\cos \left(z^{2} \pi / 2\right)$, we see that the extrema are at $z=\sqrt{2 n}$, with $n$ integer. Thus, the condition $\sqrt{2 n}=\sqrt{t} \nu \kappa$ yields the envelope pe$\operatorname{riod} \tau_{e}$ :

$$
\tau_{e}=\frac{4}{\nu^{2} \kappa^{2}} .
$$

The fast current oscillations are a consequence of Blochlike oscillations in each of the 2-state systems ( $k$-state fermions and their $k-\pi$ backscattered partners) described by Eq. (6). These arise from the $\cos (2 t-T) V_{0}$ terms in

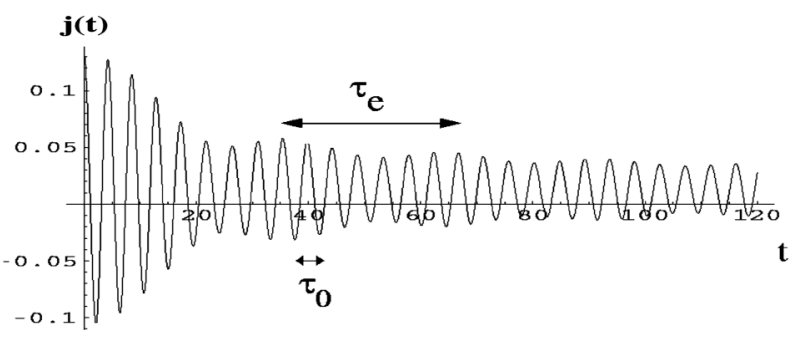

FIG. 2. Current evolution for an abrupt quench, $T=0$. We set superlattice strength $V_{0}=0.7$, hopping $w=1$, and the boost $\nu=\frac{\pi}{15}$ [i.e., initial supercurrent of $j(0) \sim 2 / 15$ ].
Eq. (14), and thus have period $\tau_{B}=\frac{\pi}{V_{0}}$. The Bloch oscillations decohere over time due to the spread in frequencies (11) of the 2-level systems. Indeed, the current in Eq. (14) eventually decays, and no current is left in the system, to lowest order in $\nu$. This decay should not be confused with decoherence due to coupling with an external bath. Rather, it is due to interference between the different $k$ modes of the system, which oscillate with different frequencies. Thus, for a system of finite size, where the $k$ frequencies are quantized, after long enough time, the current will be revived. However, this time grows rapidly with system size and is much longer than the time shown in our numerics.

Nevertheless, even in the infinite system limit, some current survives to long times. This appears in our results at higher orders in $\nu$. By computing the current averaged over long times, $\langle\bar{j}\rangle=\lim _{\mathcal{T} \rightarrow \infty} \frac{1}{\mathcal{T}} \int_{0}^{\mathcal{T}}\langle j\rangle d t$, we show that the surviving current is proportional to $\nu^{3}$ for small supercurrents. From Eq. (12), we find that the averaged current is given exactly by

$$
\langle\bar{j}\rangle=\frac{2 w \sin (\nu) \cos \left(V_{0} T\right)}{\pi}\left(1-\frac{V_{0} \arctan \left(\frac{2 w \sin (\nu)}{V_{0}}\right)}{2 w \sin (\nu)}\right) .
$$

The leading term in $\nu$ is of order $\nu^{3}$ :

$$
\langle\bar{j}\rangle \sim \frac{8 w^{2} \nu^{3}}{3 \pi V_{0}^{2}} \cos \left(V_{0} T\right),
$$

and thus absent from the treatment leading to Eq. (14).

To complement the current evolution analysis, we next study the dynamics of the momentum distribution of the HCBs. Although the analysis of the current evolution is the same for free fermions and a Tonks-Girardeu gas, the momentum distribution of the two systems is quite different. The presence of a supercurrent is described by a shift of the Fermi step function of the free Jordan-Wigner fermions, as in Eq. (3); in terms of the bosons, the supercurrent leads to a peak in the boson momentum occupation $n_{k}=\sum_{l} e^{i k l}\left\langle b_{l}^{\dagger}(t) b_{0}(t)\right\rangle$ at the boost value, i.e., $n_{k} \propto \mid k-$ $\left.\nu\right|^{-1 / 2}$ [15]. Unfortunately, an analytic description of the evolution of $n_{k}$ is a much harder task, requiring analysis of the determinants arising in the boson correlation functions. At equilibrium, the analysis is simpler: translational invariance of the system allows application of mathematical machinery such as Szego limit theorems. Our case is much less accessible, due to the incoherent mixing between the different $k$ modes.

Using exact-diagonalization techniques (see, e.g., [10]) to monitor the time evolution of the boson momentum distribution and the current, we investigated lattice sizes of up to 350 sites. The momentum distribution indeed reflects the supercurrent Bloch oscillations, as can be seen in the bosonic $n_{k}$ plotted in Fig. 3. The period of these oscillations of the peak in $n_{k}$ between $\nu$ and $\nu-\pi$ agrees very well with the analytical result $\frac{\pi}{V_{0}}$. We also confirmed numerically the main results of this manuscript, i.e., the current survival after a quench, Eqs. (17) and (18). Our 


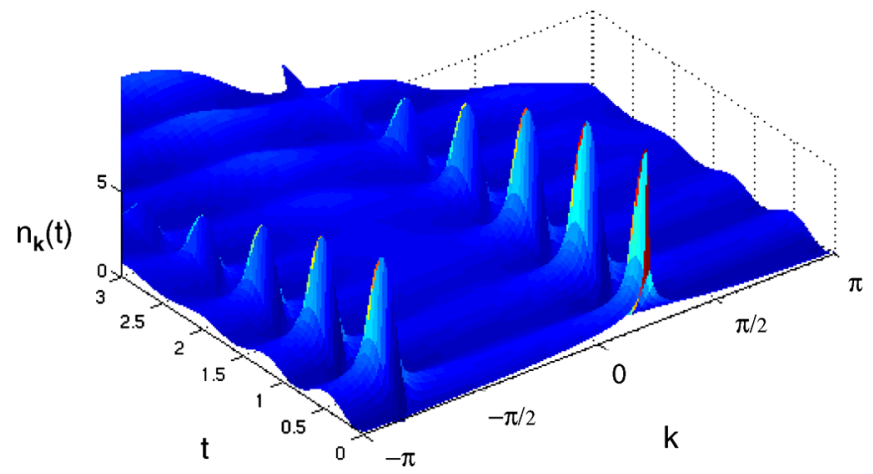

FIG. 3 (color online). $\quad n_{k}$ as a function of $k$ and time for a boost of $\nu=\pi / 5$, superlattice strength $V_{0}=4$, and hopping $w=1$. The initial peak is at $k=\nu$, and the peak transports back and forth to $-4 \pi / 5$ before disappearing due to decoherence. Results obtained using an exact diagonalization study of chains 150 sites long.

results for the long-time averaged current (in a chain of 350 sites) vs supercurrent boost $\nu$ and superlattice strength $V_{0} / w$ for an abrupt quench are summarized in Fig. 4. Perfect agreement is obtained between the analytical and numerical results.

In this Letter, we studied the behavior of hard-core bosons under a Rosen-Zener quench in the presence of a supercurrent. We described the evolution of the supercurrent, and its long-time survival fraction, as well as the corresponding momentum distribution evolution. Perhaps the most readily accessible result is the " $\nu^{3}$ law": starting with supercurrent $\nu$, the surviving current in the insulating phase is $\propto \nu^{3}$ for $\nu \ll 1$. By using the Wigner-Jordan transformation, we essentially mapped the HCB gas to a Fermi system with backscattering at the Luther-Emery line [21]. In light of our results, it is particularly interesting to ask what happens when we consider a Luttinger liquid (describing either fermions or bosons): is the $\nu^{3}$-law universal, or does it depend on the Luttinger parameter, $g \leq$ 2 ? This question, as well as a test of our predictions and the level of their universality, can be taken on experimentally.
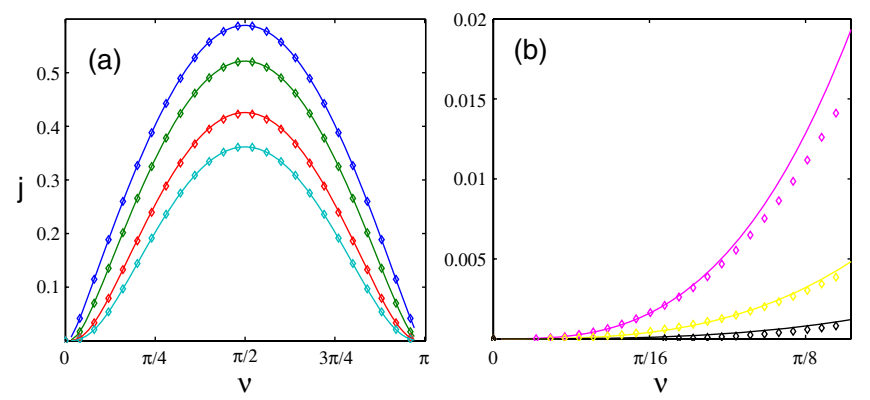

FIG. 4 (color online). Supercurrent surviving through a sudden quench. The symbols are the result of exact diagonalization of a chain 350 sites long. The hopping, $w$, is set to unity. (a) Current survival over the full range of boosts. From top to bottom, $V_{0} / w=0.1,0.25,0.5,0.7$, and the solid lines are Eq. (17). (b) The $\nu^{3}$ behavior of current survival at small currents. From top to bottom, $V_{0} / w=2,4,8$. The solid lines are Eq. (18).
This could be done, for instance, by probing cold-atoms in a ring-shaped trap, with a superimposed optical lattice. Such a geometry was recently discussed in Ref. [22]. If the additional optical lattice is made to rotate as it turns on, a supercurrent will exist in the rotating frame. The insulating phase can then be accessed by introducing a corotating superlattice. Such an experimental setup will also be able to probe nonequilibrium quenches well beyond the regimes which we considered here analytically.

We would like to thank R. Santachiara and S. Powell for discussions.

[1] A. K. Tuchman, C. Orzel, A. Polkovnikov, and M. A. Kasevich, Phys. Rev. A 74, 051601 (2006).

[2] L. E. Sadler, J. M. Higbie, S. R. Leslie, M. Vengalattore, and D. M. Stamper-Kurn, Nature (London) 443, 312 (2006).

[3] A. Maniv, E. Polturak, and G. Koren, Phys. Rev. Lett. 91, 197001 (2003).

[4] T. W. B. Kibble, J. Phys. A 9, 1387 (1976); W. H. Zurek, Nature (London) 317, 505 (1985).

[5] M. Greiner, I. Bloch, O. Mandel, T. W. Hänsch and T. Esslinger, Phys. Rev. Lett. 87, 160405 (2001); T. Stöferle, H. Moritz, C. Schori, M. Khl, and T. Esslinger, Phys. Rev. Lett. 92, 130403 (2004).

[6] B. Paredes, A. Widera, V. Murg, O. Mandel, S. Flling, I. Cirac, G. V. Shlyapnikov, T. W. Hänsch, and I. Bloch, Nature (London) 429, 277 (2004); T. Kinoshita, T. Wenger, and D. S. Weiss, Science 305, 1125 (2004).

[7] R. W. Cherng and L. S. Levitov, Phys. Rev. A 73, 043614 (2006).

[8] E. Altman and A. Auerbach, Phys. Rev. Lett. 89, 250404 (2002); A. Polkovnikov, S. Sachdev, and S. M. Girvin, Phys. Rev. A 66, 053607 (2002).

[9] A. Polkovnikov, E. Altman, E. Demler, B. Halperin, and M. D. Lukin, Phys. Rev. A 71, 063613 (2005).

[10] M. Rigol and A. Muramatsu, Phys. Rev. A 70, 031603(R) (2004); 72, 013604 (2005).

[11] P. Calabrese and J. Cardy, Phys. Rev. Lett. 96, 136801 (2006).

[12] K. Sengupta, S. Powell, and S. Sachdev, Phys. Rev. A 69, 053616 (2004).

[13] R. Schützhold, M. Uhlmann, Y. Zu, and U. R. Fischer, Phys. Rev. Lett. 97, 200601 (2006).

[14] L. Tonks, Phys. Rev. 50, 955 (1936); M. Girardeau, J. Math. Phys. (N.Y.) 1, 516 (1960).

[15] A. Lenard, J. Math. Phys. (N.Y.) 7, 1268 (1966).

[16] M. Olshanii, Phys. Rev. Lett. 81, 938 (1998).

[17] N. Rozen and C. Zener, Phys. Rev. 40, 502 (1932).

[18] E. Lieb, T. Shultz, and D. Mattis, Ann. Phys. (N.Y.) 16, 407 (1961).

[19] One may also include dissipation, which amounts to taking $c \rightarrow c+\frac{\gamma T}{\pi}$, where $\gamma$ is related to the decay of the higher energy level, which we shall ignore here [20].

[20] R. T. Robiscoe, Phys. Rev. A 17, 247 (1978).

[21] A. Luther and V. J. Emery, Phys. Rev. Lett. 33, 589 (1974).

[22] D.W. Hallwood, K. Burnett, J.A. Dunningham, arXiv:quant-ph/0609077; A. M. Rey, K. Burnett, I. I. Satija, and C. W. Clark, Phys. Rev. A 75, 063616 (2007). 